\title{
ON THE VANISHING PROBLEM OF STRING CLASSES
}

\author{
KATSUHIKO KURIBAYASHI
}

(Received 5 October 1994; revised 5 January 1995)

Communicated by J. A. Hillman

\section{Dedicated to Professor Seiya Sasao on his 60th birthday}

\begin{abstract}
The ordinary string class is an obstruction to lift the structure group $L S p i n(n)$ of a loop group bundle $L Q \rightarrow L M$ to the universal central extension of $L \operatorname{Spin}(n)$ by the circle. The vanishing problem of the ordinary string class and generalized string classes are considered from the viewpoint of the ring structure of the cohomology $H^{*}(M ; \mathbb{R})$.
\end{abstract}

1991 Mathematics subject classification (Amer. Math. Soc.): primary 57R20; secondary 55P35.

\section{Introduction}

Let $M$ be a simply connected finite dimensional manifold. Suppose that $\xi: P \rightarrow M$ is an $S O(n)$-bundle. To define the Dirac operator, we need a lifting of the structure group $S O(n)$ of $\xi$ to $\operatorname{Spin}(n)$. The bundle $Q \rightarrow M$ obtained from such a lifting is called a spin structure of $\xi$. It is well-known that a spin structure exists if and only if the second Stiefel-Whitney class $w_{2}(P) \in H^{2}(M ; \mathbb{Z} / 2)$ vanishes. Let $L M$ denote the space of smooth loops on $M$. Killingback in [4] has generalized the above conception from the viewpoint of objects on the loop space $L M$ and has shown that the generalization can be understood in terms of physical objects. In particular, the generalization of the Dirac operator in string theory has been given. When the frame bundle of $F \rightarrow M$ has a spin structure $\tilde{F} \rightarrow M$, the generalized operator is defined under the condition that the structure group $L \operatorname{Spin}(n)$ of the bundle $L \tilde{F} \rightarrow L M$ lifts to a central extension of $L \operatorname{Spin}(n)$ by the circle. In [4], the obstruction to lifting the structure group $L \operatorname{Spin}(n)$ of $L Q \rightarrow L M$ to a central extension of $L \operatorname{Spin}(n)$ has

(C) 1996 Australian Mathematical Society 0263-6115/96 \$A2.00+0.00 
been also considered, where $Q \rightarrow M$ is a spin structure for $\xi$. The obstruction has been defined by applying sheaf cohomology theory. In [5], McLaughlin has proved that $\xi$ has a spin structure if and only if the structure group $L S O(n)$ of the principal bundle $L \xi: L P \rightarrow L M$ is reducible to the identity component ([5, Proposition 2.1]). Moreover, he has also clarified results of Killingback by defining the string class $\mu(Q) \in H^{3}(L M ; \mathbb{Z})$. The class is the obstruction, which is defined without using sheaf cohomology theory, to lifting the structure group $L \operatorname{Spin}(n)$ of $L Q \rightarrow L M$ to $\hat{L S p i n}(n)$, where $\mathbb{T} \rightarrow \hat{L S} \operatorname{pin}(n) \rightarrow L \operatorname{Spin}(n)$ is the universal central extension by the circle and $n \geq 5$. One of the main theorems in [5] is as follows.

THEOREM A. ([5, Theorem 3.1.]) Let $M$ be a simply-connected, finite-dimensional manifold. The string class $\mu(Q)$ vanishes if $p_{1}(\xi) / 2$ vanishes, where $p_{1}(\xi)$ denotes the first Pontrjagin class of $\xi$. The converse is also true if $\pi_{2}(M)=0$.

The question arises whether the converse holds when the manifold $M$ is not 2connected, in particular, when $M$ is a complex Grassmann manifold. We will prove the following.

THEOREM 1. Suppose that $H^{4}(M ; \mathbb{Z})$ is torsion free and $\operatorname{dim} H^{2}(M ; \mathbb{R}) \leq 1$. Then $p_{1}(\xi) / 2$ vanishes if the string class $\mu(Q)$ vanishes.

Let $G$ be a linear Lie group and $\xi$ a $G$-bundle over $M$. In [1], Asada has defined the $p$ th string class $\tilde{C}^{p}(L \xi)$ of the $L G$-bundle $L \xi$ which belongs to the cohomology group $H^{2 p+1}(L M ; \mathbb{C})$. (Practically speaking, the higher string classes are defined for any element of the first non-abelian de Rham set of a manifold with respect to the Lie algebra of $G$ ([1, p. 11]).) Moreover it has been shown that the higher string classes have property similar to that of the ordinary string class $\mu$. The property is stated as follows.

THEOREM B. ([1, Theorem 3.3.]). For any $G$-bundle $\xi$ over $M$,

$$
\tilde{C}^{p}(L \xi)=-(2 \pi \sqrt{-1})^{p+1} p ! \int_{S^{1}} \cdot \operatorname{ev}^{*}\left(\mathrm{Ch}^{p+1}(\xi)\right),
$$

where $\mathrm{Ch}^{p+1}(\xi) \in H^{2(p+1)}(M ; \mathbb{C})$ is the $p+1$ th Chern character of $\xi$, ev : $S^{1} \times$ $L M \rightarrow M$ is the evaluation map and $\int_{S^{1}}: H^{*}\left(S^{1} \times L M ; \mathbb{C}\right) \rightarrow H^{*-1}(L M ; \mathbb{C})$ is the integration along $S^{1}$.

Thus we see that the $p$ th string class $\tilde{C}^{p}(L \xi)$ vanishes if $\mathrm{Ch}^{p+1}(\xi)=0$. In order to obtain a necessary and sufficient condition on the vanishing of string classes, we assume that 
(1.1) $H^{*}(M ; \mathbb{R})$ is isomorphic to a GCI-algebra below degree $2 s$ for some integer $s>0$. To be exact,

$$
H^{*}(M ; \mathbb{R})^{\leq 2 s} \cong\left\{\Lambda\left(y_{1}, \ldots, y_{l}\right) \otimes \mathbb{R}\left[x_{1}, \ldots, x_{n}\right] /\left(\rho_{1}, \ldots, \rho_{m}\right)\right\}^{\leq 2 s}
$$

as an algebra, where $\rho_{1}, \ldots, \rho_{m}$ are decomposable elements in the polynomial algebra $\mathbb{R}\left[x_{1}, \ldots, x_{n}\right]$ with the property that each $\rho_{i}$ is a non-zero divisor in the quotient of the polynomial algebra by the ideal generated by $\rho_{1}, \ldots, \rho_{i-1}$.

Let $\Lambda$ be the algebra $\Lambda\left(y_{1}, \ldots, y_{l}\right) \otimes \mathbb{R}\left[x_{1}, \ldots, x_{n}\right]$. For a subset $S$ of $\Lambda,(S)$ will denote the ideal of $\Lambda$ generated by $S$. We will obtain theorems on the vanishing problem of higher string classes.

THEOREM 2. Suppose that $H^{*}(M ; \mathbb{R})$ is a tensor product of truncated polynomial algebras and exterior algebras. Then, for any $p, \tilde{C}^{p}(L \xi)=0$ if and only if $\mathrm{Ch}^{p+1}(\xi)=0$.

THEOREM 3. Assume that $p \leq s-1$ and (1.1) holds. Then the pth string class $\tilde{C}^{p}(L \xi)$ vanishes if and only if $\partial \mathrm{Ch}^{p+1}(\xi) / \partial z$ belongs to the ideal $\left(\partial \rho_{j} / \partial z, \rho_{j}, ; 1 \leq\right.$ $j \leq m)$ for any $z \in\left\{x_{1}, \ldots, x_{n}, y_{1}, \ldots, y_{l}\right\}$.

We prove Theorems 1,2 and 3 by reducing to a problem of the injectivity of some derivation from $H^{*}(M ; \mathbb{R})$ to the Hochschild homology of a minimal model of the de Rham complex $\Omega(M)$. Moreover, Theorem 3 will be proved by considering when the image under the derivation of the Chern character is zero.

The author would like to thank Professor A. Asada for helpful conversation.

\section{Reduction to an algebraic problem}

From the argument of the proof of [5, Theorem 3.1] and the assumption that $H^{4}(M ; \mathbb{Z})$ is torsion free, we see that, in order to prove Theorem 1 , it suffices to consider the injectivity of the map $\int_{S^{1}} \cdot \mathrm{ev}^{*}$ to $H^{3}(L M ; \mathbb{R})$ from $H^{4}(M ; \mathbb{R})$. Since the tensor product of real cohomology and $\mathbb{C}$ preserves injectivity of a map between real cohomologies, by Theorem B, it follows that the proof of Theorem 2 can be reduced to a problem of the injectivity of the map $\int_{S^{1}} \cdot \mathrm{ev}^{*}$ between real cohomologies. Moreover, the assertion of Proposition 2.1 stated below enables us to reduce the proof of Theorems 1 and 2 to that of injectivity of the derivation $\beta^{*}[2,1.4$; p.55]. By Theorem B and Proposition 2.1, the problem of the vanishing of $\tilde{C}^{p}(L \xi)$ will be changed into that of $\beta^{*}\left(\mathrm{Ch}^{p+1}(\xi)\right)$. By making use of this fact, we prove Theorem 3. Before we describe our key proposition, we recall the definitions of the chain complex $[2,1.4]$ which is used to calculate Hochschild homology explicitly, and the iterated integral map 
[3]. Let $\Lambda V$ be a free algebra generated by a graded vector space $V=\bigoplus_{n \geq 0} V_{n}$. We define a vector space $\bar{V}$ by demanding that $\bar{V}_{n-1}=V_{n}$. For any free differential graded algebra (free DGA) $(\Lambda V, d)$, we define the free DGA $(\Lambda(V+\bar{V}), \delta)$ as follows :

(i) $\beta$ is the unique derivation of degree -1 extending the identity map $V \rightarrow \bar{V}$, and $\beta(\bar{V})=0$. We denote $\beta(v)$ by $\bar{v}$ for any $v \in V$.

(ii) $\delta$ is the unique derivation of degree +1 which satisfies that $\left.\delta\right|_{V}=d$ and $\delta \beta+\beta \delta=0$.

In [2], $\beta$ has been defined as the derivation of degree +1 for a DGA endowed with a differential of degree -1 . However, since our DGA has its differential of degree +1 , we define $\beta$ as the derivation of degree -1 .

Secondly, we recall the definition of the chain map $\sigma: \mathbb{N}(\Omega(M)) \rightarrow \Omega(L M)$ which is called the iterated integral map ([3]), where $\Omega(M)$ and $\Omega(L M)$ are the de Rham complexes of the manifolds $X$ and $L X$ respectively, and $\mathbb{N}(\Omega(M))$ is the Hochschild complex of $\Omega(M)$. Let $\varphi_{t}(t \in \mathbb{T})$ be the circle action on $L X$, generated by the vector field $T$, and $\iota$ the interior product with $T$. Let $e_{t}: L X \rightarrow X$ denote the evaluation map at time $t$. The iterated integral map $\sigma: \mathbb{N}(\Omega(X)) \longrightarrow \Omega(L X)$ is defined by

$$
\sigma\left(\omega_{0}, \ldots, \omega_{k}\right)=\int_{\Delta_{k}} \omega_{0}(0) \wedge \iota \omega_{1}\left(t_{1}\right) \wedge \cdots \wedge \iota \omega_{k}\left(t_{k}\right) d t_{1} \cdots d t_{k},
$$

where $\Delta_{k}$ is the $k$-simplex $\left\{\left(t_{1}, \ldots, t_{k}\right) \in \mathbb{R}^{k} \mid 0 \leq t_{1} \leq \cdots \leq t_{k} \leq 1\right\}$ and $\omega(t)=e_{t}^{*} \omega$. Note that the iterated integral map induces an isomorphism of algebras on cohomology ([3, Theorem 3.1, Proposition 4.1]).

PROPOSITION 2.1. (i) Let $H^{*}(\mathbb{N}(\Omega(M))$, b) be the Hochschild homology ([3]) of the de Rham complex of $M$. Then the map $\alpha: H^{*}(M ; \mathbb{R}) \rightarrow H^{*-1}(\mathbb{N}(\Omega(M)), b)$ defined by $x \mapsto(1, x)$ is a derivation of degree -1 . That is, $\alpha$ satisfies $\alpha(x y)=$ $(1, x) \cdot y+(-1)^{|x|} x \cdot(1, y)$, where $\cdot$ is the product in the Hochschild homology.

(ii) The following diagram is commutative:

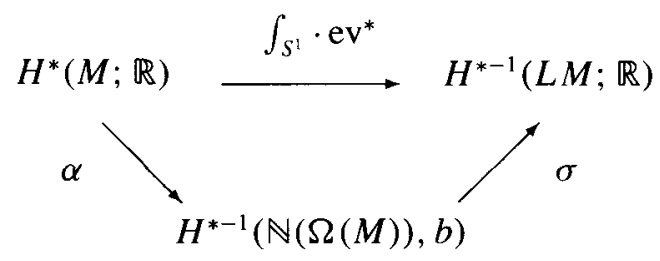

where $\sigma$ is the isomorphism induced from the iterated integral map. 
(iii) Let $\varphi:(\Lambda W, \partial) \rightarrow(\Omega(M), d)$ be a minimal model. We have the following commutative diagram:

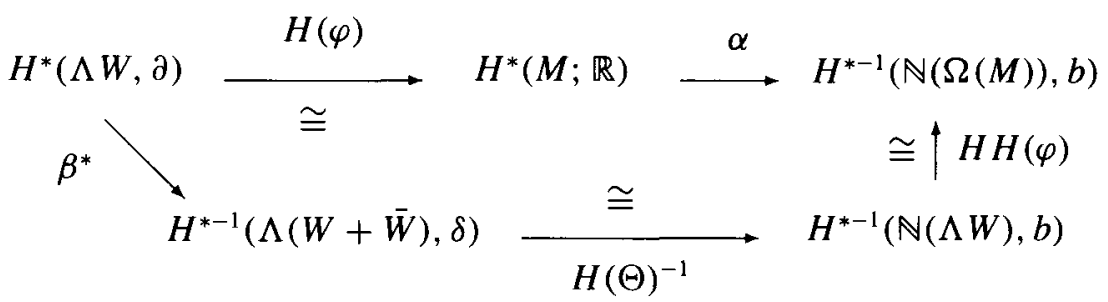

where the map $\beta$ is the unique derivation of degree -1 defined by $\beta(x)=\bar{x}$ for any base $x$ of $W$, which is a generator of the minimal model $\Lambda W$, and the map $\Theta$ which induces the isomorphism $H(\Theta)$ is defined by $\Theta\left(a_{0}, a_{1}, \ldots, a_{p}\right)=a_{0} \beta\left(a_{1}\right) \cdots \beta\left(a_{p}\right) / p$ ! ([2, Theorem 2.4]).

PROOF. By definition of the Hochschild boundary $b$, we have

$$
b(1, x, y)=-(x, y)-(-1)^{|x|-1}(1, x y)+(-1)^{(|x|-1)(|y|-1)}(y, x) .
$$

This fact implies that

$$
\begin{aligned}
(1, x y) & =(-1)^{(|x|-1)|y|}(y, x)-(-1)^{|x|-1}(x, y) \\
& =(1, x) \cdot y+(-1)^{|x|} x \cdot(1, y)
\end{aligned}
$$

in $H^{*}(\mathbb{N}(\Omega(M)), b)$. Therefore we can conclude that $\alpha$ is a derivation. Statement (ii) follows from the definition of the iterated integral map ([3]). We obtain (iii) from the definition of $\Theta$.

The assumption (1.1) enables us to construct an explicit minimal model of $\Omega(M)$ below degree $2 s-1$.

LEMMA 2.2. Suppose that (1.1) holds. Let $\Lambda V$ be the DGA

$$
\Lambda\left(y_{1}, \ldots, y_{l}\right) \otimes \mathbb{R}\left[x_{1}, \ldots, x_{n}\right] \otimes \Lambda\left(\tau_{1}, \ldots, \tau_{m}\right)
$$

whose differential $\partial$ is defined by $x_{i} \mapsto 0, y_{j} \mapsto 0$ and $\tau_{k} \mapsto \rho_{k}$. Then there exists $a$ minimal model $(\Lambda W, \partial)$ of $\Omega(M)$ such that $\Lambda V \subseteq \Lambda W$ and $\Lambda V^{\leq 2 s-1}=\Lambda W^{\leq 2 s-1}$ as $a D G A$. 
PROof. Since $H^{*}(M ; \mathbb{R})$ is a GCI-algebra below degree $2 s$, by [6, Proposition 1.1], it follows that $\Lambda V$ is a minimal model of $\Omega(M)$ below degree $2 s-1$. By extending the complex $\Lambda V$, we can obtain a minimal model of $\Omega(M)([7, \S 5])$.

By Lemma 2.2, we see that $(\Lambda(V+\bar{V}), \delta)$ is a sub-DGA of $(\Lambda(W+\bar{W}), \delta)$ and that $(\Lambda(W+\bar{W}), \delta)^{\leq 2 s-2}$ is equal to $(\Lambda(V+\bar{V}), \delta)^{\leq 2 s-2}$. Therefore we have the inclusion $H^{*}(\Lambda(V+\bar{V}), \delta)^{\leq 2 s-1} \hookrightarrow H^{*}(\Lambda(W+\bar{W}), \delta)^{\leq 2 s-1}$ and the following commutative diagrams :

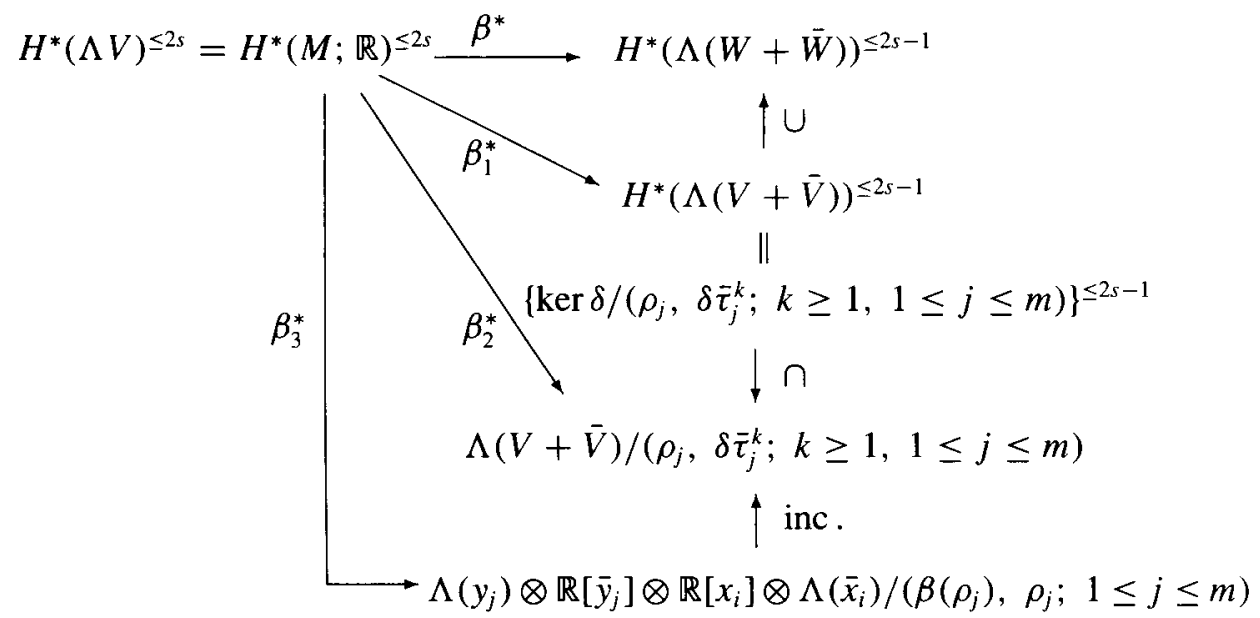

where, for any $l, \beta_{l}$ is the map defined by $x \mapsto \beta(x)$. Note that the inclusion inc. is defined since the ideal $\left(\beta\left(\rho_{j}\right), \rho_{j}\right)$ is equal to $\left(\rho_{j}, \delta \bar{\tau}_{j}^{k}\right) \cap \Lambda\left(y_{j}\right) \otimes \mathbb{R}\left[\bar{y}_{j}\right] \otimes \mathbb{R}\left[x_{i}\right] \otimes \Lambda\left(\bar{x}_{i}\right)$.

From the above argument, the problem of the injectivity of $H(\beta)$ can be reduced to a purely algebraic problem. We prepare to state this problem.

Let $\Gamma$ be a commutative algebra which is isomorphic to the algebra $\Lambda / I$ below degree $2 s$, where $\Lambda=\Lambda\left(y_{1}, \ldots, y_{l}\right) \otimes \mathbb{R}\left[x_{1}, . ., x_{n}\right]$ and $I$ is the ideal generated by elements $\rho_{1}, \ldots, \rho_{m}$ (regularity of the sequence is not assumed) of $\mathbb{R}\left[x_{1}, \ldots, x_{n}\right]$. For any element $\lambda \in \Lambda,[\lambda]_{i}$ means the sum of the term of $\lambda$ with the element $x_{i}$. Let $Q\left(\check{x}_{i}\right)$ denote an element of $\Lambda$ which consists of terms without the element $x_{i}$. Suppose that

(2.1) Any element $\left[\int \lambda \rho_{j} d x_{i}\right]_{i}$ with degree below $2 s$ belongs to the ideal $I$, where $\int$ means a formal integration and $\lambda \in \Lambda$.

(2.2) If $Q\left(\check{x}_{i}\right)=Q\left(\check{x}_{j}\right)$ in $\Lambda / I$ for any $j$, then $Q\left(\check{x}_{i}\right) \in \Lambda\left(y_{1}, \ldots, y_{l}\right)$ modulo $I$.

The injectivity of $\beta^{*}$ is shown from the following lemma. 
LEMMA 2.3. Under the assumptions (2.1) and (2.2), the map

$$
\beta_{3}^{*}: \Gamma^{\leq 2 s} \rightarrow \Lambda\left(y_{j}\right) \otimes \mathbb{R}\left[\bar{y}_{j}\right] \otimes \mathbb{R}\left[x_{i}\right] \otimes \Lambda\left(\bar{x}_{i}\right) /(\beta I, I)
$$

is injective.

The proof of Lemma 2.3 is postponed to the following section.

We describe an example of an algebra which satisfies the conditions (2.1) and (2.2).

EXAMPLE 2.4. Let $\rho_{j}(1 \leq j \leq m)$ be monomials of $\mathbb{R}\left[x_{1}, \ldots, x_{n}\right]$. The algebra $\Gamma=\Lambda / I$ satisfies the conditions. In fact, it is clear that $\Gamma$ satisfies (2.1). We can choose the element $\tilde{Q}\left(\check{x}_{i}\right)$ whose terms do not include the monomials $\rho_{j}$ as a representation of $Q\left(\check{x}_{i}\right)$ in $\Gamma$. Suppose that $Q\left(\check{x}_{i}\right)=Q\left(\check{x}_{j}\right)$ in $\Gamma$. We see that $\tilde{Q}\left(\check{x}_{i}\right)-\tilde{Q}\left(\check{x}_{j}\right) \in I$. Since all terms of element of $I$ include some $\rho_{j}$ it follows that $\tilde{Q}\left(\check{x}_{i}\right)-\tilde{Q}\left(\tilde{x}_{j}\right)=0$. Thus each term of $\tilde{Q}\left(\check{x}_{i}\right)$ does not include the element $x_{j}$, either. Thus we can conclude that $\tilde{Q}\left(\check{x}_{i}\right)$ belongs to $\Lambda\left(y_{1}, \ldots, y_{l}\right)$. So $Q\left(\check{x}_{i}\right) \equiv \tilde{Q}\left(\check{x}_{i}\right) \in$ $\Lambda\left(y_{1}, \ldots, y_{l}\right)$.

\section{Proof of Theorems 1,2 and 3}

Let $\partial / \partial z$ be the unique derivation defined by $z \mapsto 1$ and $w \mapsto 0$ if $w \neq z$, where $z \in\left\{x_{1}, \ldots, x_{n}, y_{1}, \ldots, y_{l}\right\}$. For any element $u \in \Lambda$, we will write $u$ so that the generators $y_{j}$ precede $x_{i}$ in the each term.

PROOF OF THEOREM 3. Suppose that $\partial \mathrm{Ch}^{p+1}(\xi) / \partial z$ belongs to $\left(\partial \rho_{j} / \partial z, \rho_{j}, ; 1 \leq\right.$ $j \leq m)$ for any $z \in\left\{x_{1}, \ldots, y_{1}, \ldots\right\}$. Since

$$
\beta_{3}^{*}(u)=\sum_{i} \frac{\partial u}{\partial x_{i}} \bar{x}_{i}+\sum_{j} \frac{\partial u}{\partial y_{j}} \bar{y}_{j}
$$

for any $u \in H^{*}(M ; \mathbb{C})^{<2 s}$, it follows that $\beta_{3}^{*}\left(\mathrm{Ch}^{p+1}(\xi)\right)=0$, so $\beta^{*}\left(\mathrm{Ch}^{p+1}(\xi)\right)=0$. By Proposition 2.1 (ii), we can conclude that the $p$ th string class $\tilde{C}^{p}(L \xi)$ vanishes. If $\beta^{*}(u)=0$ for some element $u \in H^{*}(M ; \mathbb{C})^{\leq 2 s}$, then $\beta_{3}^{*}(u)=0$. By definition of $\beta_{3}$, we have that

$$
(\beta(I), I) \ni \beta_{3}(u)=\sum_{i} \frac{\partial u}{\partial x_{i}} \bar{x}_{i}+\sum_{j} \frac{\partial u}{\partial y_{j}} \bar{y}_{j}
$$

Therefore we can write $\sum_{i} \frac{\partial u}{\partial x_{j}} \bar{x}_{i}=\sum_{i}\left(\sum_{j} \gamma_{j} \frac{\partial \rho_{j}}{\partial x_{i}}\right) \bar{x}_{i}+\sum_{i}\left(\sum_{j} \tilde{\gamma}_{i j} \rho_{j}\right) \bar{x}_{i}$. This implies that

$$
\frac{\partial u}{\partial x_{i}}=\sum_{j} \gamma_{j} \frac{\partial \rho_{j}}{\partial x_{i}}+\sum_{j} \tilde{\gamma}_{i j} \rho_{j} \quad \text { for any } i
$$


In consequence, the element $\partial u / \partial x_{i}$ has to belong to the ideal $\left(\partial \rho_{j} / \partial x_{i}, \rho_{j} ; 1 \leq j \leq\right.$ $m$ ) of $\Lambda$. Moreover, from this fact and (3.1), we obtain that $\sum_{j} \partial u / \partial y_{j} \bar{y}_{j} \in(\beta I, I)$. Hence the element $\partial u / \partial y_{j}$ is in $I$. Thus we have Theorem 3.

PROOF OF THEOREM 2. It suffices to prove that $\beta_{3}^{*}$ is a monomorphism. We see that the algebra $H^{*}(M ; \mathbb{R})$ satisfies the conditions (2.1) and (2.2) from Example 2.4. Theorem 2 follows from Lemma 2.3 .

PROOF OF THEOREM 1. Since $M$ is simply-connected and $\operatorname{dim} H^{2}(M ; \mathbb{R}) \leq 1$, it follows that the algebra $H^{*}(M ; \mathbb{R})$ is isomorphic, below degree 4 , to the algebra

$$
\Lambda\left(y_{1}, \ldots, y_{l}\right) \otimes \mathbb{R}\left[z, x_{1}, \ldots, x_{n}\right] /\left(\epsilon z^{2}\right)
$$

as an algebra, $\operatorname{deg} y_{j}=3, \operatorname{deg} x_{i}=4, \operatorname{deg} z=2$ and $\epsilon=0$ or 1 . Therefore $H^{*}(M ; \mathbb{R})$ satisfies the conditions (1.1), (2.1) and (2.2). We obtain Theorem 1 by Lemma 2.3.

In the case $\operatorname{dim} H^{2}(M ; \mathbb{R}) \geq 2$, from Example 2.4 we see that the consequence of Theorem 1 is true if the relations of degree 4 in $H^{*}(M ; \mathbb{R})$ are monomials and $H^{4}(M ; \mathbb{Z})$ is torsion free.

ProOF OF LemMa 2.3. Suppose that $\beta_{3}^{*}(u)=0$. From (3.2), it follows that

$$
u=\sum_{j}\left\{\gamma_{j} \rho_{j}-\left[\int \frac{\partial \gamma_{j}}{\partial x_{i}} \rho_{j} d x_{i}\right]_{i}+\left[\int \tilde{\gamma}_{i j} \rho_{j} d x_{i}\right]_{i}\right\}+Q\left(\check{x}_{i}\right)
$$

for some $Q\left(\check{x}_{i}\right)$. From (2.1), we see that $Q\left(\check{x}_{i}\right)=Q\left(\check{x}_{j}\right)$ in $\Lambda / I$ for any $i$ and $j$. By making use of (2.2), we can write $u=u_{1}+u_{2}$, where $u_{1} \in I$ and $u_{2} \in \Lambda\left(y_{1}, \ldots, y_{i}\right)$. From the equality (3.1), we obtain that $\sum_{i}\left(\partial u_{1} / \partial x_{i}\right) \bar{x}_{i}+$ $\sum_{j}\left(\partial u_{1} / \partial y_{j}\right) \bar{y}_{j}+\sum_{j}\left(\partial u_{2} / \partial y_{j}\right) \bar{y}_{j} \in(\beta(I), I)$. Since terms of an element of $(\beta(I), I)$ include some element $x_{i}$, it follows that $\sum_{j}\left(\partial u_{2} / \partial y_{j}\right) \bar{y}_{j}=0$. Therefore we can conclude that $u_{2}=0$ and that $u=0$ in $\Lambda / I$.

Let $Q \rightarrow M$ be a $\operatorname{Spin}(n)$-bundle. Finally, we consider the string class of the induced bundle $i^{*} L Q \rightarrow \Omega M$ by the natural inclusion $i: \Omega M \rightarrow L M$.

REMARK. We define a string structure for $i^{*} L Q \rightarrow \Omega M$, in a similar fashion to $L Q \rightarrow L M$, as a lifting of the structure group to $\tilde{L S p i n}(n)$ which is a non-trivial central extension of $L \operatorname{Spin}(n)$ by the circle. In particular, we take notice of the string structure for $i^{*} L Q \rightarrow \Omega M$ which is defined as a lifting of the structure group to the universal central extension $\hat{L S} \operatorname{pin}(n)$. By considering the Serre exact sequence for the bundle $i^{*} L Q \rightarrow \Omega M$, we can obtain the obstruction to defining the string 
structure as the transgression image of the generator of $H^{2}(\operatorname{LSpin}(n) ; \mathbb{Z})$ (see [5, §3]). Therefore it follows that the obstruction is $i^{*} \mu(Q)$, where $\mu(Q)$ is the ordinary string class of $Q \rightarrow M$. For any element $x$ with bar degree 0 in $H^{*}(\mathbb{N}(\Omega(M)), b)$, we see that $\sigma(x)=e_{0}^{*}(x)$, where $\sigma$ is the iterated integral map and $e_{0}: L M \rightarrow M$ is the evaluation map at time 0. Therefore, from Proposition 2.1 (i) and (ii), we can conclude that $i^{*} \mu(Q)$ vanishes if $H^{3}(\Omega M ; \mathbb{Z})$ is torsion free and there is no indecomposable element of degree 4 in $H^{*}(M ; \mathbb{R})$. In consequence, $i^{*} L Q \rightarrow \Omega M$ has a string structure under the same assumption. We also see that $i^{*} \tilde{C}^{p}(L \xi)=0$ if $H^{*}(M ; \mathbb{R})$ does not have an indecomposable element with degree $2(p+1)$.

\section{References}

[1] A. Asada, Four lectures on geometry of loop group and non Abelian de Rham theory (Chalmers University of Technology/The University of Göteborg, 1990).

[2] D. Burghelea and M. Vigué-Poirrier, Cyclic homology of commutative algebras I, Lecture Notes in Math. 1318 (Springer, Berlin, 1988), 51-72.

[3] E. Getzler, J. D. S. Jones and S. Petrack, 'Differential form on loop spaces and the cyclic bar complex', Topology 30 (1991), 339-371.

[4] T. P. Killingback, 'World sheet anomalies and loop geometry', Nucl. Phys. B 288 (1987), 578-588.

[5] D. A. McLaughlin, 'Orientation and string structures on loop space', Pacific J. Math. 155 (1992), 143-156.

[6] L. Smith, 'Homological algebra and the Eilenberg-Moore spectral sequence', Trans. Amer. Math. Soc. 129 (1967), 58-93."

[7] D. Sullivan, 'Infinitesimal computations in topology', Inst. Hautes Études Sci. Publ. Math. (1978), 269-331.

Department of Applied Mathematics

Okayama University of Science

1-1 Ridai-cho, Okayama 700

Japan

e-mail: kuri@geom.fc.ous.ac.jp 